\title{
Effect of Kinesio Taping on Hand Function in Hemiparetic Patients
}

\author{
Jonathan Galvão Tenório Cavalcante1, Maria do Desterro Costae Silva², \\ Jessiane Tenório da Fonseca Silva1, Clarissa Cotrim dos Anjos², \\ Renata Sampaio Rodrigues Soutinho ${ }^{3 *}$
}

\author{
${ }^{1}$ School of Physical Therapy, Alagoas State University of Health Sciences (UNCISAL), Maceió, Brazil \\ ${ }^{2}$ Alagoas State University of Health Sciences (UNCISAL), Maceió, Brazil \\ ${ }^{3}$ CESMAC University, Maceió, Brazil \\ Email: jonathan-gtc@bol.com.br, mdesterro@bol.com.br, siane_tenorio@hotmail.com, clacotrimanjos@gmail.com, \\ *renatasampaio_@hotmail.com
}

How to cite this paper: Cavalcante, J.G.T. do Desterro Costae Silva, M., da Fonseca Silva, J.T., dos Anjos, C.C. and Soutinho, R.S.R. (2018) Effect of Kinesio Taping on Hand Function in Hemiparetic Patients. World Journal of Neuroscience, 8, 293-302. https://doi.org/10.4236/wjns.2018.82023

Received: March 6, 2018

Accepted: May 19, 2018

Published: May 22, 2018

Copyright $\odot 2018$ by authors and Scientific Research Publishing Inc. This work is licensed under the Creative Commons Attribution International License (CC BY 4.0).

http://creativecommons.org/licenses/by/4.0/

\begin{abstract}
Introduction: Cerebrovascular accident (CVA) is the second most frequent cause of death in the world. Nevertheless, most victims do survive and need treatment, and hand function is one that has to be dealt with in the rehabilitation process. Kinesio ${ }^{\circledR}$ Taping $(\mathrm{KT})$ is a bandaging method that can be applied along the muscle fibers to provide stimulation. Studies have shown its efficacy in providing afferent stimuli to weakened muscles, thus eliciting contraction with greater recruiting of motor units and inducing neuroplasticity. Benefits to the paresthesia hand have not been reported. Objective: Investigate the effects of KT on hand function in hemiparetic patients. Material and Methods: An evaluator-blinded, randomized clinical trial involving stroke victims was carried out in a physical therapy outpatient clinic. One group underwent KT intervention and the other was a control group. The Modified Ashworth Scale (MAS) and the Box and Block Test (BBT) were used as assessment tools. A data entry form was used in the Epi-info 7 software and descriptive statistics was thus calculated. The software BioStat 5.0 was employed when doing statistical tests. Associations were regarded as statistically significant when $\mathrm{p}<0.05$. Results: Eight individuals were randomly assigned to two groups. All those who had received treatment with KT had spasticity improved by one point, but there was no significant improvement in BBT. Conclusion: KT was effective when it came to improving spasticity and it may be an option in rehab, but it had no effect on gross manual dexterity. Nevertheless, it can be of help as part of a functional training program.
\end{abstract}




\section{Keywords}

Stroke, Rehabilitation, Physical Therapy, Kinesio ${ }^{\circledR}$ Taping, Hands

\section{Introduction}

The World Health Organization has announced that stroke was the cause of 6.7 million deaths in 2012, having ranked as the second leading cause of death worldwide [1]. Yet, most victims survive. According to a study conducted in a hospital in Natal-RN, Brazil, the prevalence of lethality, as to the type of stroke, ischemic, intracerebral hemorrhage, and subarachnoid hemorrhage was 10.2\%, $17.2 \%$, and $36 \%$, respectively [2]. Another study in Brazil showed that over three decades (1982 to 2002) there was a reduction in the risk of death from the disease, a common trend in developing regions [3].

The most affected blood vessel is the middle cerebral artery [4], whose surface branches supply most of the blood to the upper-lateral parts of the cerebral cortex, an area responsible for functions such as somesthesia, speech, and motor function. Likely consequences: aphasia, hypoesthesia, and contralateral paresis, mainly in the upper limbs. The disease can be more severe if branches of the vessel deeper into the brain are affected, causing injury to basal ganglia and the internal capsule [5].

It is a challenge for motor rehabilitation programs to find a way to stimulate the sensorimotor system in order to re-establish functional voluntary movement [6]. Some treatment options are: Bobath Method, Kabath, Functional Electrical Stimulation, Music Therapy, and Constraint-Induced Movement Therapy [7] [8] [9]. However, the efficacy of these methods remains to be determined by further studies.

The Kinesio ${ }^{\circledR}$ Taping Method was devised in the 1970s by Doctor Kenzo Kase. He used the Kinesio ${ }^{\circledR}$ Tex Tape, a latex free cotton material which possesses the proper degree of elasticity, adherence, and breathability. The technique can be used to retrain the nervous system, ease pain, improve physical performance, prevent injuries, and promote circulation and regeneration [10].

Some studies have argued that Kinesio ${ }^{\circledR}$ Taping is effective on weakened muscles, for it generates continuous afferent stimuli to the central nervous system due to the fact that the bandage is in prolonged contact with the skin. Such stimuli would be able to elicit muscle response with greater recruiting of motor units and to aid in neuroplasticity [11] [12].

Kinesio ${ }^{\circledR}$ can be used on the paresthesia upper limb to improve scapular alignment, reduce glenohumeral subluxation and the resulting joint pain [6]. No studies were found showing the use of Kinesio ${ }^{\circledR}$ Taping in the rehabilitation of the paresthesia hand in patients who had stroke sequelae, particularly with regard to better spasticity and improvement in range of motion (ROM) relating to the active extension of the fingers (metacarpophalangeal and interphalangeal 
joints).

Hence, this study was aimed at investigating the effects of Kinesio Taping ${ }^{\circledR}$ as part of hand rehabilitation for patients with hemiparesis, and the results were assessed using the Modified Ashworth Scale (MAS) and the Box and Block Test (BBT).

\section{Methods}

A blind randomized clinical trial was carried out at the Professor Delza Gitaí outpatient clinic (CEF) of the school of physical therapy at the Alagoas State University of Health Sciences-UNCISAL. For purposes of defining the sample, the total number of stroke patients admitted and waiting list totaling 10 patients was evaluated.

All individuals undergoing physical therapy were randomly assigned to two groups: the Kinesio group (KG) and the control group (CG). The control group performed conventional physical therapy conducted by undergraduated physical therapy students under the supervision of a physical therapist specialist in neurorehabilitation. It was comprised $40 \mathrm{~min} /$ day, 2 days/week. Therefore, only the KG patients were treated with Kinesio Taping.

Inclusion criteria were as follows: 18 years or older, both genders, paresis of an upper limb (UL) due to stroke, and undergoing physical therapy. Exclusion criteria were: unhindered function of both upper limbs, diagnosis of other neurological disorders, amputation, dermal or osteomyoarticular injury of the UL that may impair treatment, and vulnerable and uncooperative patients.

Medical records were examined and patients and health care professionals were questioned. Those eligible were asked to sign the Informed Consent Form and were then interviewed and made aware of the procedures. On the BBT, those whose performance was equal to or greater than 43 blocks per minute were excluded because, according to a chart with results for individuals aged 25 to 75-plus, their numbers fell within the expected range for healthy people [13].

A form was filled out with information on gender, date of birth, dominant side, month and year of CVA and affected hemisphere, and the recorded results of the MAS and BBT. Lastly, possible physiotherapeutic treatments for the paresthesia hand were also recorded. Each physiotherapy session had been routinely added to the patients' medical records, thus allowing for a comparison of the rates of use of this therapeutic approach for both groups. Also, orthoses and botulinum toxin were used.

The assessments were conducted before and after the 15 physical therapy treatment sessions, and the Kinesiology Tape (KT) was applied in 12 of these sessions (difference was established to cover for unforeseen cases in which contact with the patient was not possible). The assessments happened before each physical therapy treatment session was begun in order to avoid the influence of any of the physical therapy tools used. For the KG group, reevaluation was conducted three to seven days after removing the KT tape. 
Kinesio $^{\oplus}$ Tex Tape was placed on the anterior surface of the forearm, hand, and fingers down to the intermediate phalanges with a $25 \%$ to $35 \%$ tension. Proximal and distal anchor points (no tension) were, respectively, the lateral epicondyle and the distal phalanges (Figure 1). The tape was cut after measuring the distance from right above the lateral epicondyle, down the forearm and all the way to the metacarpo-phalangeal joints. After that, the tape was measured from the end of the 3rd finger to the middle third of the 3rd metacarpal bone and then folded onto itself. Finally, three longitudinal cuts were made all the way to the folded end, thus resulting in four strips, one for each of the fingers (2nd to 5 th).

During the clinical trial, care was provided twice a week at the outpatient clinic, and some patients even came once a week. All patients were seen by physical therapy interns under the supervision of skilled neurological physiotherapy specialists.

The Modified Ashworth Scale (MAS) was used to assess spasticity. MAS is an ordinal 6-point scale that rates spasticity as follows: $0=$ no increase in muscle tone; 1 = slight increase in muscle tone with minimal resistance at the end of the range of motion; $1+=$ slight increase in muscle tone, followed by minimal resistance in less than half of the ROM; $2=$ more marked increase in muscle tone throughout most of the ROM, but affected part(s) easily moved; 3 = considerable increase in muscle tone, passive movement difficult; $4=$ Affected part(s) rigid in flexion or extension [14]. Wrist and finger flexor muscles' resistance to passive

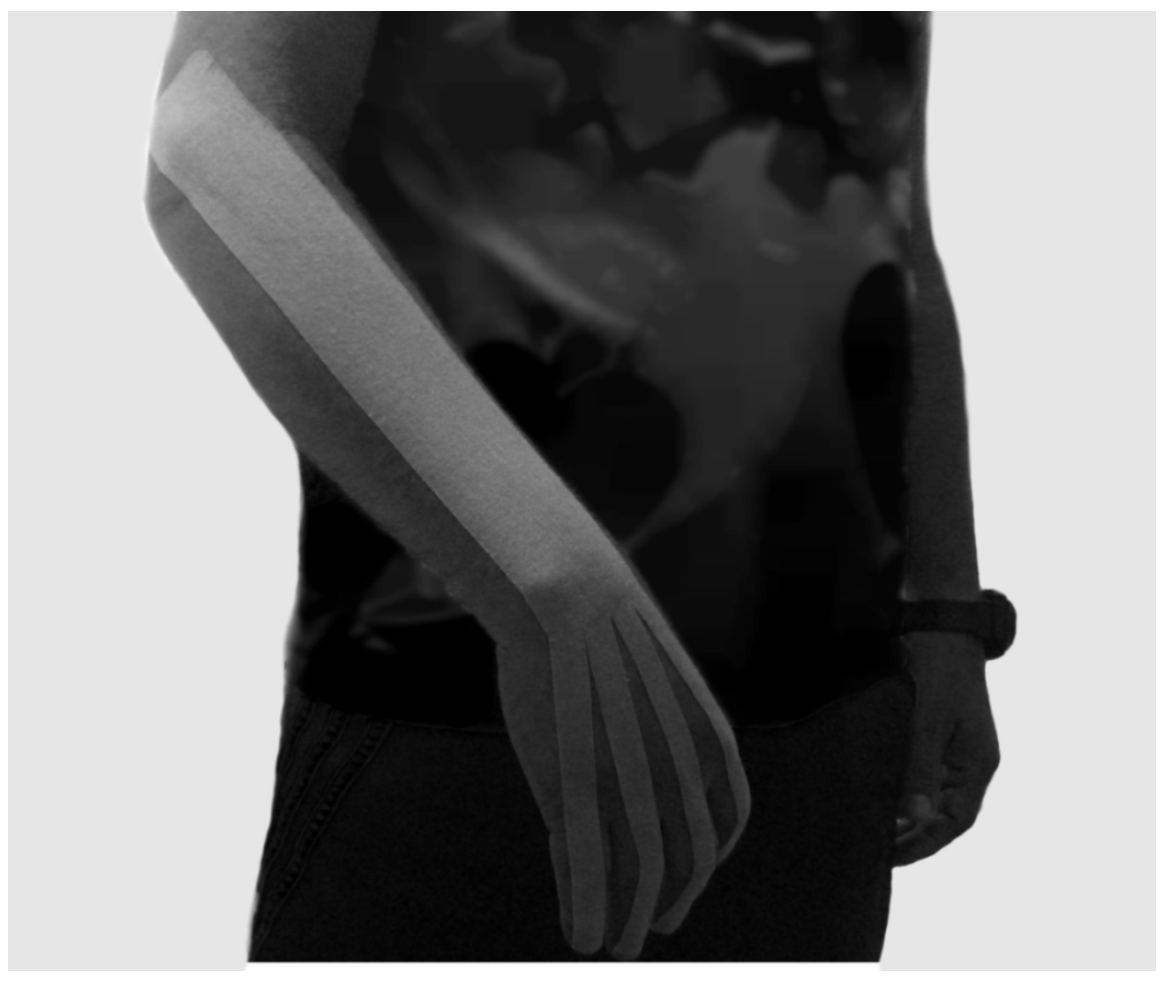

Figure 1. Applying Kinesio Taping to aid the extensor muscles of the wrist and fingers. Source: Effects of Kinesio Taping on hand function in hemiparetic patients. 
stretch was measured having each individual comfortably seated and with the upper limb at rest and the wrist flexed [15].

In order to evaluate function at the extremity of the upper limb, the Box and Block Test (BBT) was used to measure gross manual dexterity [16]. The test consists of a wooden box divided into two compartments by a partition and with all 150 blocks placed on one side. The person is asked to move, one by one, the maximum number of blocks from one compartment of the box to the other of equal size. The result of the test is the number of blocks moved within 1 minute (bpm). The material was manufactured and used in accordance with adult norms [13].

The assessment was carried out by a single blinded examiner in order to reduce inter-examiner variability and preference bias for one group over the other.

Data were entered into forms created using the Epi-info 7.0 software, which was also used to calculate descriptive statistics. The statistical tests were run on the BioStat 5.0 software. Distribution normality was assessed using the Lilliefors Test and homogeneity of variance as well as the difference between the means on the t-test for independent samples. The Mann-Whitney test (non-parametric) shall be used if none of the assumptions that justify the use of the parametric $\mathrm{t}$-test are true. The binomial test was conducted to compare how often spasticity improved between the groups. The association was deemed statistically significant when $\mathrm{p}<0.05$.

This study was approved by the Research Ethics Committee under protocol 17,267,913.0.0000.5011.

\section{Results}

Medical record sheets were assessed for 21 patients who had been admitted to the neurological physiotherapy center and for 9 who had been on a waiting list. All patients had suffered a CVA. Two patients who had been on the waiting list were enrolled into the trial while data were being gathered. Overall, the losses totaled 16 patients; one died, eight were given outpatient discharge, and seven were waiting for the beginning of the treatment; six were excluded because they showed no signs of upper limb extremity impairment. Ultimately, eight individuals were enrolled, four assigned to each group.

Patients in the KG had a mean age of $42.0 \pm 1.41$, whereas for those in the CG mean age was $59.0 \pm 19.64$, which meant normality within the groups, but heteroscedasticity was present (unequal variance, $\mathrm{p} \leq 0.005$ ). Therefore, an alternative nonparametric test was used, which indicated a non-significant difference between the means $(p=0.24)$. As to the time from the moment of the CVA to the day of the interview, the average for the KG was 44.75 months \pm 41.07 and $57.25 \pm 41.56$ for the CG, thus attaining normality, homoscedasticity, and non-significant difference between the means ( $\mathrm{p}=0.68$ with the $\mathrm{t}$-test).

The results from the MAS showed a 1-point improvement in the score of spasticity in $100 \%$ of the valid individuals $(n=3)$ of the KG, except for that per- 
son who initially had MAS 0 . As for the CG, the same 1-point improvement occurred in $25 \%$ of the sample $(n=1)$. The difference was deemed significant $(\mathrm{p}=$ 0.02 )

Results from the BBT showed improvement for just one individual in each group, thus making it impossible to statistically compare the groups. By grouping all individuals according to the hemisphere affected, we were able to see better performance before and after treatment for those patients with left hemiparesis (mean $7.75 \pm 5.73$ upon first evaluation and $10.0 \pm 8.04$ on second testing), when compared to those with right hemiparis (the same initial and final mean score of $1.0 \pm 1.41)$. Nevertheless, the Mann-Whitney test result was not significant, $\mathrm{p}=0.14$ (initial and final). The other tests showed data normality and heterogeneity.

Table 1 shows full characterization of the individuals and assessment results.

Treatment options were assessed. Only those methods/tools used specifically on hand and wrist were considered, both post-assessment and pre-reassessment of patients. Data are arranged in such a way as to show, for a certain number of sessions, how many times those methods/tools were employed. It is important to observe that patient s2 of CG and patient s4 of KG were seen fewer times because of one individual's absenteeism record and late treatment enrollment of the other (Table 2).

Stretching was the most commonly used therapeutic option, with average utilization at $7 \pm 6.05$ for the KG and at $10.7 \pm 4.9$ for the CG, there being normality, homoscedasticity, and no significant difference $(\mathrm{p}=0.24)$. By using all other data found in Table 2, it was not possible to arrive at representative averages to compare the two groups.

Table 1. Group characterization and assessment of individuals who had cerebrovascular accident. Maceió, AL, 2014.

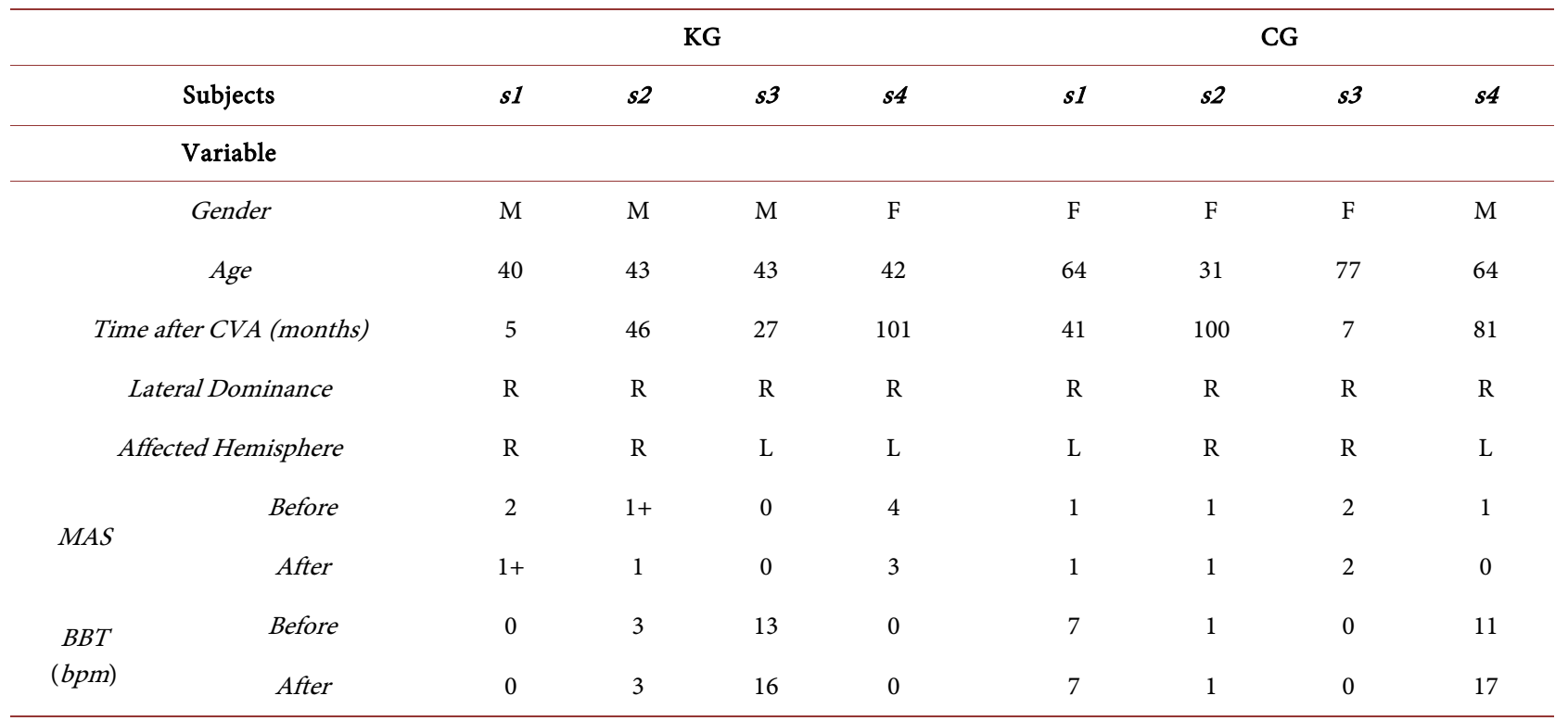

CVA: CerebroVascular Accident; KG: Kinesio Group; CG: Control Group; M: Male; F: Female; R: Right; L: Left; MAS: Modified Ashworth Scale; BBT: Box and Block Test; bpm: blocks per minute. Source: effects of Kinesio Taping on Hand Function in Hemiparetic Patients. 
Table 2. Percent use of therapeutic resources in the rehabilitation of the paresthesia hand in patients who had cerebrovascular accident. Maceió, AL, 2014.

\begin{tabular}{|c|c|c|c|c|c|c|c|c|}
\hline \multirow[b]{2}{*}{ Subjects } & \multicolumn{4}{|c|}{ KG n (\%) } & \multicolumn{4}{|c|}{ CG n (\%) } \\
\hline & $S 1$ & $S 2$ & $S 3$ & $S 4$ & $S 1$ & $S 2$ & $S 3$ & $S 4$ \\
\hline No of sessions & 15 & 15 & 15 & 6 & 15 & 6 & 15 & 15 \\
\hline \multicolumn{9}{|l|}{ Therapeutic resource } \\
\hline Stretching & $8(53.3)$ & $15(100)$ & $1(6.6)$ & $4(66.6)$ & $15(100)$ & $6(100)$ & $7(46.6)$ & $15(100)$ \\
\hline Active/assisted ROM & & $1(6.6)$ & $3(20.0)$ & & $2(13.3)$ & & $1(6.6)$ & $1(6.6)$ \\
\hline Passive ROM & & $2(13.0)$ & & & & $2(33.3)$ & $3(20.0)$ & \\
\hline Cryotherapy & $2(13.3)$ & & & $2(33.3)$ & & & & \\
\hline Weight bearing & $3(20.0)$ & $2(13.0)$ & & & & & & $1(6.6)$ \\
\hline Electrical stimulation & & $9(60.0)$ & $1(6.6)$ & & & & & $1(6.6)$ \\
\hline Manual stimulation & & $3(20.0)$ & & $1(16.6)$ & $3(20.0)$ & $1(16.6)$ & & $1(6.6)$ \\
\hline Texture stimulation & & $1(6.6)$ & & $1(16.6)$ & $1(6.6)$ & $1(16.6)$ & & \\
\hline $\begin{array}{l}\text { Stretch reflex on extensor } \\
\text { muscles }\end{array}$ & & $1(6.6)$ & & & $2(13.3)$ & $1(16.6)$ & & $1(6.6)$ \\
\hline
\end{tabular}

KG: Kinesio Group; CG: Control Group; S: subject; N: number; ROM: Range Of Motion. Source: Effects of Kinesio Taping on Hand Function in Hemiparetic Patients.

An individual in the KG used a wrist orthosis only in public settings or when using public transport and no one had underwent recent procedure or put through a procedure during the research regarding the use of botulinum toxin.

\section{Discussion}

Sensorimotor impairment caused by lesions to the left and right Middle Cerebral Arteries (MCA) was investigated by Voos and Do Vale [17], who showed that, when impaired, the right side (as a result of left-side hemiparesis) had a better rate of recovery. The BBT seems to corroborate what was found by the said authors, thus pointing to better performance by individuals with right hemisphere injury. Poor results on the BBT by individuals who had their left hemisphere affected can be explained by the fact that the latter is more involved in motor skills that require planning [18].

Historically, it was believed that the reduction in spasticity would lead to an improvement in motricity [19]. This hypothesis has been revisited because, when spasticity is inhibited, function does not return to patients who suffered a CVA.

One of the two groups that underwent standard physiotherapy treatment was given botulinum toxin injections, whereas the other one received a placebo. The comparison of the two groups showed no significant difference in active work performed with the upper limb [20]. Similarly, in the case of cerebral palsy in both children and adults, the patient who underwent treatment to reduce spasticity showed no improvement when it came to controlling his or her movements [21]. 
Based on what has been stated, it is worth asking why individuals who scored better on the BBT had a lower degree of spasticity. This is likely related to less extensive or less severe lesions of the corticospinal tract as well as of premotor areas and supplementary motor areas [22], thus resulting in better residual motor function. However, once intentional movement becomes impossible, it is unlikely that only a treatment to reduce spasticity and which does not include a motor relearning program can result in considerable functional gains.

The results obtained made it impossible to state or suggest that there is a relation between the degree of spasticity and the injured cerebral hemisphere. However, in one other study that also did not validate such a hypothesis and in which MAS was not used, only the presence or not of hypertonia was assessed [22].

All individuals in the KG who had suffered from spasticity benefited from the 1-point reduction as measured by the Modified Ashworth Scale (MAS). Statistical comparison revealed that, when associated with physical therapy and as demonstrated in this paper, the use of KT to facilitate flexion of the extensor muscles of wrist and fingers is more effective than the use of KT on its own.

Washiet et al. [23] showed that proprioceptive afferents contribute to the sense of self, but that for such the signals from cutaneous receptors are not essential. Furthermore, external stimuli do cause changes to neural circuits [24]. In addition to stimulating encapsulated nerve endings of the skin, it is possible that KT stimulates proprioceptors by modifying both articular positioning and the length of agonist and antagonist muscles.

Based on the above-mentioned authors, the physiological effects of KT in improving spasticity may be in the cortical reorganization attributed to the new articular positioning caused by KT tension against the upper limb flexor pattern, thus contributing to better results as measured by MAS. However, it is necessary to investigate the role played by cutaneous receptors in ameliorating not only paresis but also sensibility disturbances, which were the object of this study.

The common extensor muscle of the fingers is frequently affected and its activation is important to counteract flexion of the wrist and fingers, which is commonly seen in many patients [25]. According to the results of a study by Slupik et al. [12], the use of KT in healthy individuals increases the tone of the treated muscles. The author suggests that the improved muscle tone was the result of greater recruitment of motor units, but that its underlying cause is not yet clear. It is likely that this effect may also occur in muscles affected by diseases of the central nervous system such as CVA, and that the utilization of KT to promote greater activation of the extensor muscles may inhibit flexor spasticity.

\section{Conclusions}

Kinesio Taping, when associated with physiotherapy treatment, was effective in reducing spasticity of the wrist and fingers in patients with post-CVA hemiparesis. Although KT did not induce recovery of hand motor function, one cannot rule out the fact that it may be helpful as part of a functional treatment program. 
Upon completion of this study, it is possible to recommend the inclusion of KT in the list of physical therapy tools for the treatment of hemiparesis caused by a cerebrovascular accident and also to suggest the reanalysis of physiotherapeutic treatment for rehabilitation, such as in the case of hand function.

This study had some limitations which have to be pointed out: the small sample size, which may have a negative impact on the validation of the statistical tests, the difference in age between the groups in the study, and the lack of a physiotherapy treatment protocol. There was also an individual on whom Kinesio Taping had been applied fewer times, but whose results were the same as those of individuals treated twice as many times. This fact raises concerns as to the number of times Kinesio Taping should be applied to generate lasting reduction in spasticity and as to the duration of those effects. It is hoped that further studies may contribute to a better understanding of the benefits of Kinesio Taping and of the challenges in cerebrovascular accident rehabilitation.

\section{References}

[1] WHO (World Health Organization) (2014) The Top 10 Causes of Death. Fact Sheet $\mathrm{N}^{\circ}$ 310. http://www.who.int/mediacentre/factsheets/fs310/en/

[2] Martins, A.N.N.J., Figueiredo, M.M., Rocha, O.D., Fernandes, M.A.F., Jerônimo, S.M.B. and Dourado, M.E.J. (2007) Frequency of Stroke Types at an Emergency Hospital in Natal, Brazil. Arq Neuropsiquiatr, 65, 1139-1143.

https://doi.org/10.1590/S0004-282X2007000700009

[3] André, C., Curioni, C.C., Cunha, C.B. and Veras, R. (2006) Progressive Decline in Stroke Mortality in Brazil from 1980 to 1982, 1990 to 1992, and 2000 to 2002. Stroke, 37, 2784-2789. https://doi.org/10.1161/01.STR.0000244768.46566.73

[4] Ng, Y.S., Stein, J., Ning, M. and Black-Schaffer, R.M. (2007) Comparison of Clinical Characteristics and Functional Outcomes of Ischemic Stroke in Different Vascular Territories. Stroke, 38, 2309-2314. https://doi.org/10.1161/STROKEAHA.106.475483

[5] Machado, A. (2000) Functional Neuroanatomy. 2nd Edition, Editora Atheneu, São Paulo, 92.

[6] Jaraczewska, E. and Long, C. (2006) Kinesio ${ }^{\circledR}$ Taping in Stroke: Improving Functional Use of the Upper Extremity in Hemiplegia. Topics in Stroke Rehabilitation, 13, 31-42. https://doi.org/10.1310/33KA-XYE3-QWJB-WGT6

[7] Mikołajewska, E. (2012) NDT-Bobath Method in Normalization of Muscle Tone in Post-Stroke Patients. Advances in Clinical and Experimental Medicine, 21, 513-517.

[8] Oujamaa, L., Relave, I., Froger, J., Mottet, D. and Pelissier, J.Y. (2009) Rehabilitation of Arm Function after Stroke. Literature Review. Annals of Physical and Rehabilitation Medicine, 52, 269-293. https://doi.org/10.1016/j.rehab.2008.10.003

[9] Villeneuve, M. and Lamontagne, A. (2013) Playing Piano Can Improve Upper Extremity Function after Stroke: Case Studies. Stroke Research and Treatment, 2013, Article ID: 159105. https://doi.org/10.1155/2013/159105

[10] Kase, K., Wallis, J. and Kase, T. (2003) Clinical Therapeutic Applications of the Kinesio Taping ${ }^{\oplus}$ Method. 2nd Edition, Kinesio Taping Association, Dallas, 12.

[11] Santos, J.C.C., Giorgetti, M.J.S., Torello, E.M., Meneghetti, C.H.Z. and Ordenes, I.E.U. (2010) The Influence of Kinesio Taping in the Treatment of Shoulder's Subluxation at Stroke. Rev Neurocienc, 18, 335-340. 
[12] Slupik, A., Dwornik, M., Bialoszewski, D. and Zych, E. (2007) Effect of Kinesio Taping on Bioelectrical Activity of Vastusmedialis Muscle. Ortopedia Traumatologia Rehabilitacja, 9, 644-651.

[13] Mathiowetz, V., Volland, G., Kashman, N. and Weber, K. (1985) Adult Norms for the Box and Block Test of Manual Dexterity. American Journal of Occupational Therapy, 39, 386-391. https://doi.org/10.5014/ajot.39.6.386

[14] Bohannon, R. and Smith, M. (1987) Interrater Reliability of a Modified Ashworth Scale of Muscle Spasticity. Physical Therapy, 67, 206-207. https://doi.org/10.1093/ptj/67.2.206

[15] Heidari, M., Abootalebi, S. and Hosseini, S.A. (2011) Validity of Modified Ashworth Scale as a Measure of Wrist Spasticity in Stroke Patients. Iranian Rehabilitation Journal, 10, 26-30.

[16] Salter, K., Jutai, J., Zettler, L., Moses, M., Mcclure, A., Mays, R., et al. (2012) Outcome Measures in Stroke Rehabilitation. In: The Evidence-Based Review of Stroke Rehabilitation, 54-55.

[17] Voos, M.C. and Do Valle, L.E.R. (2008) Comparative Study between the Hemisphere's Relationship to Stroke and Functional Evolution in Right-Handed Individuals. Revista Brasileira De Fisioterapia, 12, 113-120.

[18] Haaland, K.Y., Prestopnik, J.L., Knight, R.T. and Lee, R.R. (2004) Hemispheric Asymmetries for Kinematic and Positional Aspects of Reaching. Brain, 127, 1145-1158.

[19] O'dwyer, N.J., Ada, L. and Neilson, P.D. (1996) Spasticity and Muscle Contracture Following Stroke. Brain, 119, 1737-1749. https://doi.org/10.1093/brain/119.5.1737

[20] Ward, A.B., Wissel, J., Borg, J., Ertzgaard, P., Herrmann, C. and Kulkarni, J. (2014) Functional Goal Achievement in Post-Stroke Spasticity Patients: The Botox Economic Spasticity Trial (BEST). Journal of Rehabilitation Medicine, 46, 504-513. https://doi.org/10.2340/16501977-1817

[21] Neilson, P.D. and Mccaughey, J. (1982) Self-Regulation of Spasm and Spasticity in Cerebral Palsy. Journal of Neurology, Neurosurgery, and Psychiatry, 45, 320-330. https://doi.org/10.1136/jnnp.45.4.320

[22] Brown, P. (1994) Pathophysiology of Spasticity. Journal of Neurology, Neurosurgery, and Psychiatry, 57, 773-777. https://doi.org/10.1136/jnnp.57.7.773

[23] Walsh, L.D., Moseley, G.L., Taylor, J.L. and Gandevia, S.C. (2011) Proprioceptive Signals Contribute to the Sense of Body Ownership. The Journal of Physiology, 589, 3009-3021. https://doi.org/10.1113/jphysiol.2011.204941

[24] Vidyasagar, R., Folger, S.E. and Parkes, L.M. (2014) Re-Wiring the Brain: Increased Functional Connectivity within Primary Somatosensory Cortex Following Synchronous Co-Activation. NeuroImage, 92, 19-26. https://doi.org/10.1016/j.neuroimage.2014.01.052

[25] Butler, A.J., Kahn, S., Wolf, S.L. and Weiss, P. (2005) Finger Extensor Variability in TMS Parameters among Chronic Stroke Patients. Journal of NeuroEngineering and Rehabilitation, 2, 10. https://doi.org/10.1186/1743-0003-2-10 